\title{
Context Proceduralization in Decision Making
}

\author{
Jean-Charles Pomerol and Patrick Brézillon \\ LIP6, Université Pierre et Marie Curie \\ 4, Place Jussieu - 75252 Paris Cedex 05 \\ \{Jean-Charles.Pomerol, Patrick.Brezillon\}@lip6.fr
}

\begin{abstract}
Although it seems obvious that decision making is a contextual task, papers dealing with decision making tackle rarely the problem of contextual information management. After a brief presentation of our view on context, we examine the contextual dimension of decision making. Then we explain our views about the acquisition of contextual data and the construction of a reasoning framework appropriate for decision making. We call this process proceduralization and we refer to a rational construction for action (rca).
\end{abstract}

Keywords: decision making, contextual knowledge, proceduralized context.

\section{Introduction}

Starting from the view that contextual knowledge exists as the background knowledge describing a decision situation, we argue that effective action requires the extraction of a subjective part of this contextual knowledge called proceduralized context. In other words a decision problem is interpreted as a case (in the artificial intelligence sense $[14,10,11])$ and the decision maker builds a reasoning for action using some the contextual clues contained in the case. This construction is called proceduralization. In this paper, we discuss of the process of proceduralization under the light of some psychological views and experiments about decision making.

\section{Some Reminding about Context}

To understand more accurately the relationships between decision making and context we have to be more specific about what we call context. From an engineering point of view, we can start from a definition of the context as the collection of relevant conditions and surrounding influences that make a situation unique and comprehensible $[12,1]$. The difficulty with this definition is that there are "numerous interacting factors that people do not even pay attention to on a conscious level, and many of which are outside the ability of machine input devices to capture" [9]. In the above definitions the authors have clearly in mind the fact that the context is not under the control of the observer. 
Let us take an example, in the control of a subway line [7]: a large amount of knowledge about trains, electricity, people's reactions, etc. contributes to make the situation unique, while some more particular conditions about the time, the day, the weather and so on, influence decision making more specifically. In other words, there is beforehand a common background context which is then specified by some conjectural and contingent influences. For example, the general context is subway control which differs from train or bicycle control although they share some mechanical laws and the particular context is specific to a line, a day, an hour, etc. These considerations explain why Tiberghien [25] defines context as the whole set of secondary characteristics of a situation or secondary properties of a cognitive or motivational state of an individual that can modify the effect of an effective stimulation (stimulus) or an oriented activity.

Thus, it would probably be wise to talk of primary and secondary contexts to distinguish between the general, relatively fixed primary characteristics of a situation, and the secondary characteristics. If we think about primary context, we must confess that it is difficult to avoid the word knowledge about this general background used by the operators to carry out their task. These are some of the reasons why in a previous study [5], we defined three types of context. First, the context which is shared by those involved in the problem and is directly but tacitly used for the problem solving. Second, the context that is not explicitly used but influences the problem solving. Third, the context that has nothing to do with the current decision making but is known by many of those involved. We call these three types of context respectively: proceduralized context, contextual knowledge and external knowledge.

Contextual knowledge is more or less similar to what people generally have in mind about the term 'context'. It contains some general information about the situation and the environment of the problem. Contextual knowledge implicitly delimits the resolution space (this idea is also evoked in [2]). It is always evoked by a task or, an event, but does not focus on a task or on the achievement of a goal but is mobilized according to a set of tasks, even though it has not yet been proceduralized for use. Contextual knowledge is on the back-stage, whereas the proceduralized context is on the front-stage under the spotlights. It is noteworthy that, as far as engineering is concerned, only the proceduralized context matters, but contextual knowledge is necessary because this is the raw material from which proceduralized context is made. One can say that contextual knowledge is proceduralized, not necessarily explicitly, to become the proceduralized context. In a sense, the proceduralized context is the contextual knowledge activated and structured to make diagnoses, decisions and actions. This aspect is discussed in a concrete way in the framework of the contextual graphs [4].

The proceduralized context is a part of the contextual knowledge that is invoked, structured and situated according to a given focus. The proceduralized context is the part of the contextual knowledge which is proceduralized before decision but having the decision making problem in mind. According to the current decision making step, 
a piece of the contextual knowledge either enters the proceduralized context or becomes external knowledge. Thus, the content of the context evolves continuously all through the decision making. Once the first pieces of contextual knowledge are mobilized, some other pieces of contextual knowledge, such as the position of the incident on the line, also enter the focus of attention and are proceduralized. Finally, whereas the contextual knowledge is rather independent of the subject, the proceduralized context is a personal construct for action.

\section{Proceduralization}

We argued that a crucial step in dealing with context as regards decision making is the proceduralization step [5,6]. At this step, the decision maker picks into the back-stage context the data and information which he feels are necessary to make a decision in the case at hand. According to the previous discussion (section 2), the proceduralization acts as a filter upon the contextual knowledge. Actually, the process is twofold. On the one hand it consists of selecting relevant information. Thus, the proceduralized context appears as a sub-part of the whole contextual knowledge. Note that in the selection of the contextual elements for the proceduralized context, there is a part of arbitrary, and, as a consequence, a proceduralized context can be better than another one. On the other hand, the decision makers try to organize the knowledge in such way that the proceduralized context can be used for decision. The problem for the decision maker is then to build the rationales of the observed facts and, if possible, to anticipate the consequences of the possible actions. Let us call rationale construction for action (rca hereafter) the second part of the process.

Thus, the context is not only a set of objective characteristics describing a situation as is often claimed (see [24] for some usual views on context and knowledge), but the mental representation (as quoted in Cognitive Ergonomics for representing and interpreting external events) generated by the words of the description as well as the risk attitude also matter as regards decision. In other words, the proceduralization of identical facts depends on the mental representation of the decision maker that constitute a context evoked by the presentation. Presented in a context of loss or mortality, the same facts do not trigger the same decision as presented in a context of gains Tversky and Kahneman [26, 27, 28]. The reader could object that everybody knows that decision maker's mood clearly influences decision making. One can say that an optimistic person does not evaluate the consequences of an action and/or the probabilities of the events in same manner as a pessimistic one. The frame effect is seemingly different, the probabilities as well as the consequences are the same but the evaluation is changed because different visions are associated to the different representations. The difference does not come from the person's mood but from the words that describe the situation and of the brain images associated to the situation. This does not discard the necessity and the possibility to start from an objective description of the facts and an accurate diagnosis, but rises the question of 
what is an objective representation in face of representation biases ? Obviously, there is an interference with the image generated and/or recorded in the mind of a subject in the representation of a context. The sensible representation of the context depends on the experience of the subject.

The acquisition of the contextual facts is selective and is obviously sensitive to some availability and representativeness biases [15, p. 82]. It is likely that more recent or more striking events will be more easily recalled. This view is also common in cognitive science [1]. When the subject can easily get a representation of the situation because he is familiar with, the probability of occurrence and the prominence of the phenomenon have a chance to be increased (representativeness effect, see [27]). Using some prior knowledge, the subject captures a set of contextual facts that are extracted form what we called the contextual knowledge. When these facts are available, the proceduralization consists of structuring this facts in order to make them useful for decisions. The first step is to understand why the situation is that which is observed in order to anticipate the effects of possible actions. During the next step, what is important is to identify the causal and consequential links between the facts: this is the $r c a$ phase as introduced at the beginning of this section.

The $r c a$ process is even more prone to biases than selection. First, the subject can interpret positive correlation and contingency as causal rules. Moreover, it has been observed a phenomenon of search for dominance [17,18]. This search for dominance tends to justify a posteriori the choice by proving that the chosen action dominates the others. According to our view, this search for dominance is nothing else than a rationalization process either before decision or more generally after the decision. As such, when it occurs before action it is a kind of rca. Actually, the process of search for dominance is already carried out during the proceduralization because people privilege the causal and consequential links which reinforce their prior beliefs. To some extent the contextual knowledge is extracted, according to some choice which is already made, in order to justify this choice. As mentioned by Weinberger [29], to make a decision amounts to build a story that makes sense and whose the denouement is precisely the action commanded by the decision. Thus the rca process organizes the observed facts, records the ones that fits with the diagnosis on which the subject is anchored [19]. Then, continuing the story, the diagnosis opens the door to some anticipation and various scenarios. Once more, there is no decision without the capability of writing scenarios [21, 22]. This entails that the rca process is tale dependent! People very easily adhere to "good stories" even to explain purely contingent events. This is why so frequent are the beliefs of plot and/or purposeful action of powerful leaders when an accident or a catastrophe occurs (see [15, p. 83]; [20, p. 164]). In some sense, magic thought and illusory correlations [8] are frequent, this is a sign of a perverse proceduralization: people proceduralize without any evidence because they need these links to organize the "small world" structuring the decision at hand [3]. 
In a study about the management of the incidents on an underground line [7] we observed that the contextual knowledge is one of the main components of diagnosis construction. In other words, this means that the operators of the line try to gather as much as possible of contextual elements to know what is the context of the incident, because this context determines their diagnosis and the subsequent actions. We also observed that the proceduralization consists of building a diagnosis consistent with the incoming information. Moreover, the $r c a$ process also encompasses the design of some scenarios to allow an anticipation of the forthcoming events and of the results of the possible actions. In this process, the uncertainty is reduced by action postponement [22] and the prior gathering of the maximum of information. When one operator chooses to undertake an action, the uncertainty is rather about the current context than about future. In other words, it could be said that if the operator could know the exact context of his action, he could accurately anticipate its result. Thus, in the operator's decision making process, we think that the most important bias are those that are related to knowledge acquisition, namely anchoring, representativeness and availability. They can impede the proceduralization of the contextual knowledge both during the selection and the rca.

To sum up, we can say that there is a "construct" more or less rational before decision making occuring during the proceduralization of the contextual knowledge. This process is very important, it must be thoroughly checked and system supported because human beings tends to find "rational causes" even to purely contingent events. One can say that human are contingency-averse, especially for unfavorable events! It is thus necessary to bring some rationality for building scenarios and for proceduralizing the contextual knowledge. This is what we tried to do for subway control by using contextual graphs in which the user must specify the context value at each contextual node.

\section{Conclusion}

The contextual knowledge influences decision making, but data and information that are more or less shared by everybody do not influence per se. To understand the role of the contextual knowledge it is necessary to understand the cognitive process that occurs between context, apprehension and decision making. The cognitive biases shed some light on this process.

An essential characteristic of context is its dynamics. First, the understanding of external event depends of the current backstage knowledge available, but that is a knowledge that evolves. Second the decision making process (diagnosis) has also a contextual nature that depends on the decision-maker background, background that itself evolves. This is, in our viewpoint, a reason to make explicit the proceduralization process. As a side effect, there is a need for an incremental 
knowledge acquisition for improving the continuous building and rebuilding of the mental representation through the proceduralization process.

The first lesson is that the process of context management is twofold: contextual knowledge acquisition and rationale construction for action ( $r c a)$. While the first step of contextual knowledge acquisition and interpretation is subject to the cognitive biases of anchoring and availability the second step is more difficult to describe and analyze.

Context acquisition results into a list of contextual elements which are instantiated when used by somebody. In the two models we recalled in the introduction, this knowledge is easily represented by context nodes in context graphs and by criteria in multicriterion decision. However, let us observe that this last representation is poorly adapted to context use because the criteria generally denote attributes controlled or to be controlled by the decision maker whereas the most significant contextual element which are not under control and are, as such, difficult to apprehend in a multicriterion analysis framework where it turns out that actions and criteria are thought as controlled, the former by definition and the latter by intention.

Actually, the proceduralization step is difficult to model. Let us recall that during this decisive step the subject picks out data and facts in the contextual knowledge in order to build his proceduralized context. This construction is vaguely similar to the conception of a story linking facts and consequences. At this step a structuration of the knowledge occurs resulting in a meaningful organization of the world preparing action (denoted $\mathrm{rca}$ in the paper). This rationalization separates diagnosis and the contextual elements for the diagnosis of the current state from the anticipated consequences. This is a temporal process for which scenario representation is adapted and, up to now, without no other competitive representation. The result of the proceduralization step is the basis for decision making.

Unfortunately the $r c a$ process can be impeded by many biases which are described in the literature, see [23] for a survey. Among these biases are illusory correlation [8], illusion of control [13, 16] and reinforcement, all consolidating false inferences, neglecting small probabilities and ignoring unfrequent events and consequently eliminating them from scenarios [19]. There are few possibilities to obviate these cognitive biases but validating scenarios and accumulating knowledge via graphical representations. This is what we tried to promote in the underground control case. It remains that identical contextual knowledge leads to different decisions because the proceduralization process is subjective. Efforts must be done by system designers to facilitate and decrease the subjectivity of the proceduralization process. 


\section{References}

1. Anderson J.R., 1995, Cognitive Psychology and its Implications, Freeman, New York.

2. Bainbridge L., 1997, The change in concepts needed to account for human behavior in complex dynamic tasks. IEEE transactions on Systems, Man and Cybernetics, 27, 351359.

3. Berkeley D. and Humphreys P., 1982, Structuring Decision Problems and the "Bias Heuristic", Acta Psychologica 50, 201-252.

4. Brézillon P. (2003) Contextual graphs: A context-based formalism for knowledge and reasoning in representation. To appear in a Research Report, LIP6, University Paris 6, France.

5. Brézillon P. and Pomerol J-Ch., 1999, Contextual Knowledge sharing and cooperation in intelligent assistant systems, Le Travail Humain 62 (3), PUF, Paris, 223-246.

6. Brézillon P. and Pomerol J-Ch., 2001, Modeling and Using Context for System Development : Lessons from Experience, Journal of Decision Systems 10, 265-288.

7. Brézillon P., Pomerol J-Ch. and Saker I., 1998, Contextual and contextualized knowledege, an application in subway control, Special Issue on Using Context in Applications, International Journal on Human-Computer Studies 48 (3), 357-373.

8. Chapman L.J. and Chapman J.P., 1969, Illusory Correlation as an obstacle to the use of valid psychodiagnostic signs, Journal of Abnormal Psychology 74, 271-280.

9. Degler D. and Battle L., 2001, Knowledge Management in Pursuit of Performance : the Challenge of Context, http://www.pcd.innovations.com/kminpursuit/id3-m.htm.

10. Gilboa I. and Schmeidler D., 1995, Case-Based Decision Theory, Quaterly Journal of Economics 110, 605-639.

11. Gilboa I. and Schmeidler D., 2000, Case-Based knowledge and Induction, IEEE Transactions on Systems, Man and Cybernetics 30, 85-95.

12. Hasher L. and Zack R.T. (1984) Automatic processing of fundamental information : the case of frequency of occurrence, American Psychologist 39, 1372-1388.

13. Kahneman D. and Lovallo D., 1993, Timid choices and Bold Forecasts: A cognitive perspective on Risk Taking. Management Science 39, 17-31.

14. Kolodner J., 1993, Case-based Reasoning, Morgan Kaufmann, San-Francisco.

15. Leake, D B, 1991. "Goal-based explanation evaluation" Cognitive Science 15(4).

16. McKenna F.P., 1993, It won't happen to me: unrealistic optimism or illusion of control, British Journal of Psychology 84, 39-50.

17. Montgomery H., 1983, Decision rules and the search for a dominance structure : towards a process model of decision making. In P.C. Humphreys, O. Svenson and A. Vari (Eds), Analysing and aiding Decision Processes, North Holland, 343-369.

18. Montgomery H., 1987, Image theory and dominance search theory: how is decision making actually done ? Acta Psychologica 66, 221-224.

19. Morel C., 2002, Les décisions absurdes, Galimard, Paris.

20. Piattelli-Palmarini M., 1995, La réforme du jugement ou comment ne plus se tromper, Odile Jacob, Paris.

21. Pomerol J-Ch., 1997, Artificial intelligence and human decision making, European Journal of Operational Research 99, 3-25.

22. Pomerol J-Ch., 2001, Scenario Development and Practical Decision Making under uncertainty, Decision Support Systems 31, 197-204.

23. Pomerol J-Ch., 2002, Decision Making Biases and Context, Brussels DSS Conference, Journal of Decision Systems to appear.

24. Pomerol J-Ch. and Brézillon P., 2001, About some relationships between Knowledge and context, Submitted.

25. Tiberghien G., 1986, Context and Cognition: Introduction. Cahier de Psychologie Cognitive 6(2), 105-119. 
26. Tversky A. and Kahneman D., 1982a, Judgment under uncertainty: Heuristics and biases, in Judgment under uncertainty: Heuristics and biases, Kahneman D., Slovic P. and Tversky A. (Eds.), Cambridge University Press, Cambridge, U.K., 3-20.

27. Tversky A. and Kahneman D., 1982b, Subjective probability: A judgment of representativeness, in Judgment under uncertainty: Heuristics and biases, Kahneman D., Slovic P. and Tversky A. (Eds.), Cambridge University Press, Cambridge, U.K., 32-47.

28. Tversky A. and Kahneman D., 1988, Rational Choice and the Framing of Decisions, in Decision Making, Bell D.E. et al. (Eds.), 167-192.

29. Weinberger D., 2001, Garbage in, Great Staff Out, Harvard Business review $79 n^{\circ} 8,30$ 32. 